\title{
Coping with artifact in the analysis of flow cytometric data
}

\author{
Vera S. Donnenberg'1,2,3 and Albert D. Donnenberg ${ }^{2,3,4}$
}

1University of Pittsburgh School of Medicine, Dept. of Cardiothoracic Surgery

2 University of Pittsburgh Cancer Institute

3 McGowan Institute of Regenerative Medicine

4 University of Pittsburgh School of Medicine, Dept. of Medicine

Corresponding authors:

Albert D. Donnenberg, Ph.D.

Professor of Medicine

5117 Centre Avenue, Suite 2.42

Pittsburgh, Pennsylvania 15213

E-mail: donnenbergad@upmc.edu

Phone (direct): 001-412-623-3256

Vera S. Donnenberg, Ph.D.

Associate Professor of Cardiothoracic Surgery

5117 Centre Avenue, Suite 2.42

Pittsburgh, Pennsylvania 15213

E-mail: $\underline{\text { donnenbergvs@upmc.edu }}$

Phone (direct): 001-412-623-7784 


\section{Abstract}

This article highlights several sources of artifact that interfere with optimal analysis of flow cytometric data. Such problems are compounded when flow cytometry is performed on mechanically and enzymatically disaggregated solid tissues or on cultured cells, where subcellular debris, apoptotic or necrotic cells, and highly autofluorescent cells may comprise a substantial proportion of acquired events. We provide real-world examples of tissues that pose specific analytical challenges (bone marrow, breast cancer, lung cancer and adipose tissue) and suggest approaches to improve data analysis. These include the use of a sequential or hierarchical gating process, which envisions analysis as consisting of three parts: 1) Removal of artifact; 2) Defining classifying populations; and 3) Measuring outcomes on the classifying populations. Tools for removal of artifact include use of the time parameter to detect and remove fluidic perturbations, use of doublet discrimination to avoid analysis of cell clusters, measurement of DNA content to remove subcellular debris and late apoptotic cells, Boolean gating to recognize and remove autofluorescent events, and the use of a dump gate (markers known to be negative on the population of interest, but expressed on interfering cells). Implementation of these strategies, as appropriate, extends the usefulness of flow cytometry to a wider range of applications.

Keywords: Artifact, Adipose Tissue, Breast Cancer, Lung Cancer, Bone Marrow, Autofluorescence

\section{Abbreviations}

$\begin{array}{ll}\text { DAPI } & \text { 4',6-diamidino-2-phenylindole } \\ \text { DNase } & \text { Deoxyribonuclease } \\ \text { ECD } & \text { Energy coupled dye } \\ \text { FAD } & \text { Flavin adenine dinucleotide } \\ \text { FDA } & \text { Food and drug administration } \\ \text { FITC } & \text { Fluorescein isothiocyanate } \\ \text { FMO } & \text { Fluorescence minus one } \\ \text { FMOplus } & \text { Fluorescence minus outcomes } \\ \text { HBSS } & \text { Hank's balanced salt solution } \\ \text { HIV } & \text { Human immune-deficiency virus } \\ \text { IRB } & \text { Internal review board } \\ \text { ISHAGE } & \text { International society for hematotherapy and graft engineering } \\ \text { KU } & \text { Kunitz units } \\ \text { NADH } & \text { Reduced nicotinamide adenine dinucleotide } \\ \text { PBS } & \text { Phosphate buffered saline } \\ \text { PF } & \text { Phvcnervthrin }\end{array}$


PMT Photomultiplier tube

SVF Stromal vascular fraction

UPCI University of Pittburgh Cancer Institute

UPMC University of Pittburgh Medical Center

WBC White blood cells

Graphical Abstract (see figure)

\section{Introduction}

The field of flow cytometry has evolved to encompass cells and tissues from multiple sources, the simultaneous use of multiple fluorochromes, and the detection of rare cells among a very large denominator of interrogated events. Even in the best prepared samples, these applications bring with them multiple sources of artifact that can compromise analytical results unless recognized and addressed appropriately during data analysis. This article will provide three examples of difficult samples (bone marrow, solid epithelial tumors and adipose tissue), in order to demonstrate the problems and offer analytical solutions.

\section{Material and methods}

2.1. Human Samples. The bone marrow CD34 determination was performed by the UPMC Hematopoietic Stem Cell Laboratory as a diagnostic assay. The data were anonymized by an honest broker and is used under University of Pittsburgh IRB exemption (PRO14030569). The breast cancer and lung cancer samples were collected under IRB approved protocols (UPCIO4-162 and UPCI99-053, respectively). 
2.2. CD34 staining. Flow cytometry was performed using a commercially available FDA approved diagnostic assay (Stem-Kit, Beckman-Coulter Immunotech, Marseille, France, Cat. No. IM3630). All regents were included in the kit. Anti-CD45 FITC/CD34 PE (20 $\mu \mathrm{L})$ was added to 12 x 75 polystyrene tubes in duplicate. A negative control cocktail (Isoclonic control), consisting of anti-CD45 FITC/CD34 PE plus a large excess of unlabeled anti-CD34, was added to a third tube. Cell suspension (100 $\mu \mathrm{L}$ of sample) was added quantitatively using a positive displacement pipettor (model 2226 ooo-6, Eppendorf Brinkmann, Westbury, NY, USA). The tube was capped to prevent evaporation and incubated at room temperature in the dark for $20 \mathrm{~min}$. Ammonium chloride-based lysing solution (2 $\mathrm{mL})$ and $7 \mathrm{AAD}(20 \mu \mathrm{L})$ were then added. After a $10 \mathrm{~min}$ incubation at room temperature in the dark, $100 \mu \mathrm{L}$ of Stem-Count calibration beads were quantitatively added at a known concentration of 1002 beads/ $\mu \mathrm{L}$ and the tubes were held on ice. Samples were acquired on a 5-color Beckman Coulter FC500 cytometer, calibrated daily with FlowCheck and FlowSet beads (Beckman-Coulter). A wash tube was run between the two experimental tubes and the control tube to eliminate sample carryover. Three levels of process controls (CD-Chex CD34, level 1, level 2, level 3, Cat. Nos. 213336, 213346 and 213382, Streck Laboratories, Omaha NE, USA) were run with each sample as positive controls of known low intermediate and high CD34 content.

2.3. Tumor disaggregation. Single cell suspensions were prepared from freshly excised tumor tissue as previously described. Step-by-step protocols are available in $(1,2)$. Briefly, tumors and lung tissue were minced with paired scalpels and digested with type I collagenase (0.4\% in RPMI 1640 medium, Cat. No. C-0130, Sigma Chemicals, St. Louis MO) and DNase (350 KU/mL, Sigma Chemicals, St. Louis MO, Cat. No. D-5025) and disaggregated through 100 mesh stainless steel screens. Undigested tissue clumps were subjected to repeated rounds of digestion. Viable cells were separated from erythrocytes and debris on a Ficoll-Hypaque gradient (Histopaque 1077, Sigma Chemicals). Erythrocytes were lysed using an $\mathrm{NH}_{4} \mathrm{Cl}$-based lysing solution without fixative (Beckman-Coulter, Cat No. IM3630d).

\subsection{Tumor staining}

2.4.1. Prevention of nonspecific binding and surface staining. Non-specific binding of fluorochromeconjugated antibodies was minimized by preincubating pelleted cells for 5 minutes with neat 
decomplemented $\left(56^{\circ} \mathrm{C}, 30\right.$ minutes) mouse serum $(5 \mu \mathrm{L})$, centrifuged and decanted (2). In the example shown in Figures 2 and 3, prior to intracellular cytokeratin staining, cells were stained for surface markers (2 $\mu \mathrm{L}$ each added to the cell pellet, 15-30 minutes on ice; CD44-PE (BeckmanCoulter, Cat No. A32537), CD9o-biotin (BD, Cat.No. 555594), Streptavidin-ECD (Beckman Coulter, Fullerton, CA Cat. No. IM3326), CD14-PECy5 (Beckman-Coulter, Cat. No. IM2640U), CD33-PECy5 (Beckman-Coulter, Cat. No. IM2647U), Glycophorin A/CD235a-PECy5 (BD Biosciences, Cat.No.559944), CD45-APCCy7 (BD, Cat. No. 348805)), and fixed with 2\% methanolfree formaldehyde (Polysciences, Warrington, PA).

2.4.2. Intracellular staining for cytokeratin and DNA content. Gentle cell permeabilization after extracellular staining and fixation is necessary for intracellular staining. This permits DNA the intercalating dye DAPI to be used to measure DNA content, which we also used to excluding events with degraded DNA or no DNA. Surface stained, fixed cells were permeabilized with $0.1 \%$ saponin (Coulter) in phosphate buffered saline with $0.5 \%$ human serum albumin (10 minutes at room temperature), cell pellets were incubated with $5 \mu \mathrm{L}$ of neat mouse serum for 5 minutes, centrifuged and decanted. The cell pellet was disrupted and incubated with $2 \mu \mathrm{L}$ of anti-pan cytokeratin-FITC (Beckman Coulter, Cat. No. IM2356) for 30 minutes. Cells pellets were diluted to a concentration of 10 million cells $/ 400 \mu \mathrm{L}$ of staining buffer and DAPI (Life Technologies, Grand Island NY, Cat. D1306 ) was added 10 minutes before sample acquisition, to a final concentration of $7.7 \mu \mathrm{g} / \mathrm{mL}$ and $40 \mu \mathrm{L} / 10^{6}$ cells. The intensity of DNA staining depends on both the cell and dye concentrations and can be quite variable. Lymphocytes in the tissue sample were identified (Figure 2, pink region) during sample acquisition and used to adjust PMT gain, such that the brightness of the $2 \mathrm{~N}$ peak fell in a convenient fluorescence channel.

2.5. Adipose disaggregation. Single cell suspensions were prepared from whole fat tissue and lipoaspirates as previously described $(3,4)$. Briefly, fat tissue was thoroughly minced with scissors. The aqueous portion of lipoaspirate was removed by aspiration after centrifugation. The resulting fatty tissue was digested for $30 \mathrm{~min}$ in Hanks' Balanced Salt Solution (HBSS, Invitrogen) containing 3.5\% bovine serum albumin (BSA, Millipore, Charlottesville, VA) and $1 \mathrm{mg} / \mathrm{ml}$ collagenase Type II (Worthington, Lakewood, $\mathrm{NJ}$ ) on a shaking water bath at $37^{\circ} \mathrm{C}$, and finally disaggregated through successive $425 \mu \mathrm{m}$ and $180 \mu \mathrm{m}$ sieves (W.S. Tyler, Mentor, OH). Mature adipocytes were eliminated by centrifugation 
(400g, ambient temperature, $10 \mathrm{~min}$ ) and cell pellets were resuspended in $\mathrm{NH}_{4} \mathrm{Cl}$-based erythrocyte lysis buffer (Beckman Coulter, Miami, FL, Cat No. IM363od), incubated for 10 min at ambient temperature, and washed in PBS. Some samples were depleted of debris on a Ficoll-Hypaque density gradient (Histopaque1-1077, Sigma). Freshly isolated cells from the stromal vascular fraction (SVF) were maintained on ice and stained for analytical flow cytometry as previously described (5).

2.6. Adipose staining. Cell suspensions were centrifuged (200g, $7 \mathrm{~min})$ and the cell pellet was preincubated with $5 \mu \mathrm{L}$ neat mouse serum (Sigma), centrifuged and decanted, to minimize non-specific antibody binding. Cells were simultaneously stained with monoclonal mouse anti-human antibodies (CD105-FITC (Fitzgerald, Acton, MA, Cat No. 61R-CD105-DHUFT), CD73-PE (BD Biosciences, Cat No. 550257), CD146-biotin (Miltenyi Biotec, Auburn, CA, Cat No. 130-092-852), CD14-PECy5 (Beckman Coulter, Cat No. IM2640U), CD33-PECy5 (Beckman Coulter, Cat No. IM2647U), GlycophorinA/CD235a-PECy5 (BD Biosciences, Cat No. 559944), CD31-PECy7 (Biolegend, San Diego, CA, Cat No. 303117), CD9o-APC (BD Biosciences, Cat No. 559869), CD34 APC-Alexa 700 (Beckman Coulter, Cat No. A86354), and CD45-APCCy7 (BD Biosciences, Cat No. 348805), $2 \mu \mathrm{L}$ each, on ice), washed and incubated with streptavidin-PE-Texas Red (ECD, Beckman Coulter, Cat No. IM3326). Analytical samples were fixed with $2 \%$ methanol-free formaldehyde (Polysciences, Warrington, PA), permeabilized in PBS with $0.1 \%$ saponin (Coulter), $0.5 \%$ BSA for $10 \mathrm{~min}$ at ambient temperature and incubated with $7.7 \mu \mathrm{g} / \mathrm{ml}$ DAPI.

2.7. Sample acquisition and data analysis (tumor and adipose samples). Multi-dimensional flow cytometric acquisition was performed using a 10-color Gallios cytometer (Beckman Coulter, Miami FL). The cytometer was calibrated to predetermined photomultiplier target channels prior to each use using 8-peak Rainbow Calibration Particles (Spherotech, Libertyville, IL, Cat. No. RCP-30-5A). Threshold was set on DAPI fluorescence to exclude subcellular debris and up to 1.8 million events were acquired per sample. The DAPI signal was acquired independently on two violet channels optimizing voltage settings of two individual photomultiplier tubes for removal of debris and cell cycle analysis. For compensation purposes, BD CalibriteTM beads (BD Biosciences) and single antibody-stained mouse IgG capture beads (BD Biosciences) were acquired for single fluorochromes (FITC, PE, APC) and tandem-dyes (ECD, PECy5, PECy7, and APCCy7), respectively. Initially, regions and gates were set with the aid of "fluorescence minus one" isotype controls. Offline compensation and analysis were 
performed using the high throughput parallel processing VenturiOne software (Applied Cytometry, Sheffield, UK).

\section{Theory/calculation (single platform absolute counts)}

Initially, flow cytometers were not designed to measure cell concentrations in a sample. In the first applied determinations, $\mathrm{CD} 4$ counts in HIV infection, $\mathrm{CD} 4+$ cells $/ \mu \mathrm{L}$ of peripheral blood were obtained from three clinical measurements: a white blood cell count, a lymphocyte percentage (differential), and CD4+ cells quantified by flow cytometry as a proportion of the lymphocyte scatter gate. In 2003 the Centers for Disease Control introduced guidelines for performing absolute CD4 counts using a lyse/no-wash single platform method similar to that used in the present article for CD34 determinations. Absolute counts were calculated as follows (6):

Absolute Count cells per $\mu L=$ No. of events of interestNo. of calibration beads xTotal No. of beads addedSample volume $(\mu L)$

Several modern analytical cytometers (Attune NxT, Life Technologies; CytoFLEX, Beckman Coulter) have syringe based sample pumps and therefore can determine both the number of events and the sample volume interrogated in a given counting interval.

\section{Results}

\subsection{CD34 staining and the ISHAGE protocol}

Enumeration of viable $\mathrm{CD} 45^{+/ \mathrm{CD}} 34+$ hematopoietic progenitor cells has become the gold standard for hematopoietic graft quality (7). It's measurement in peripheral blood is critical to determining when to leukapherese a patient whose bone marrow has been mobilized with cytokines, and its measurement on leukapheresis, bone marrow and umbilical cord blood products is used to determine the adequacy of stem cell dose, replacing the much more time consuming hematopoietic colony forming assays as a standard, and permitting real time measurement. Unfortunately, bone marrow, the original application for $\mathrm{CD}_{34}$ determination, has several complicating factors: 1) It contains myeloid cells, some of which have high autofluorescence in the channels usually used for FITC and PE measurement due to the presence of flavonoids and other fluorescent biomolecules (8); 2) It contains abundant nucleated red blood cells which do not express CD45 and can affect the denominator; 3) CD34 is relatively weakly expressed on the cell 
surface; 4) $\mathrm{CD} 34+$ cells are relatively rare, about $1 \%$ of $\mathrm{CD} 45^{+}$events in bone marrow and well-mobilized peripheral blood (and about $0.05 \%$ in unmobilized or poorly mobilized peripheral blood); 5) CD34 viability is usually high in cellular products, but can be compromised by cryopreservation or other manipulations. Quantitative analysis of $\mathrm{CD}_{34}+$ events in an assay with known and reproducible performance characteristics is necessary for this critical analyte and a great deal of effort has been expended to standardize its measurement between clinical laboratories world-wide (9).

4.1.1.The assay. Although sample preparation is not the focus of this article, it is useful to review the principal attributes of the lyse/no-wash single-platform assay (10) that has become the standard as an FDA-approved in vitro diagnostic test. In such a system, fluorochrome-conjugated antibodies are added directly to a carefully measured sample aliquot. After the antibodies have bound, lysis buffer is added to lyse erythrocytes, 7AAD is added to stain the DNA of non-viable (permeant) cells, and an exact volume of beads (usually the same as the sample volume) are added at a known concentration. In another variant of the assay, special staining tubes are provided with a known number of beads pre-added to the tube. When the sample is acquired a ratio is calculated of the count of the events of interest (e.g. CD45+/CD34+/7AAD- events) divided by the bead count. This ratio is multiplied by the known bead concentration and yields the absolute concentration of the events of interest. Since there are no centrifugation steps, there is no possibility of cell or bead loss. The assay is almost foolproof, provided that the sample and bead volumes are measured with sufficient precision, and the sample is well-mixed. Although the persistence of unbound fluorescent antibodies in the sample decreases sensitivity somewhat, this is not apparent to the user since unbound antibody only raises the baseline above which events are measured, and does not increase the measured fluorescence intensity of cells. The assay is completed by analysis of 7AAD staining to identify permeant (non-viable) cells. This type of single-platform events has replaced dual platform assays in which the percent of positive events, as determined on a flow cytometer, is multiplied by the concentration of cells, as measured on a hematology instrument or hemocytometer. The dual platform method suffers from the wellknown denominator problem (i.e. the definition of a cell is instrument dependent) and contributes two sources of measurement error rather than one. 
4.1.2. Data analysis. Most techniques for analysis of flow cytometry data grew out of single or dual color immunological studies on peripheral blood. According to prevailing methods, light scatter (forward and side scatter) was used to identify cells and segregate them from low light scatter debris using a logical gate. The scatter-gated population was then used as the denominator upon which events of interest (positive events) were identified for "percent positive" determinations. Although this method is well suited to analysis of peripheral blood mononuclear cells, where debris and residual platelets can usually be resolved from small lymphocytes by light scatter alone, this gating strategy falls apart when debris is prevalent, or when large numbers of events have been acquired causing the debris population and cell population to visually merge in the scatter histogram. Sutherland recognized that this uncertainty, as well as the problem contributed by autofluorescent cells, were responsible for poor standardization among clinical laboratories quantifying $\mathrm{CD}_{34}+$ cells for critical clinical applications, and was the lead author on recommendations for improved $\mathrm{CD} 34$ enumeration (11). These recommendations departed radically from the conventional gating sequence, taking advantage of our a priori knowledge of about the CD34 cells. These recommendations, known as the ISHAGE method (for the International Society for Hematotherapy and Graft Engineering, later renamed the International Society for Cellular Therapy), have been adopted almost universally by the hematopoietic stem cell transplantation community.

4.1.3. Sequential gating: The ISHAGE method. The method illustrated here is the adaptation of the ISHAGE method in current use by the University of Pittsburgh Medical Center's Hematopoietic Stem Cell Laboratory. This protocol and our instrumentation quality control measures, are virtually unchanged since their inception in the early 2000's allowing unprecedented tracking of results and comparison of historical data. The sample is from a bone marrow product harvested from a healthy sibling marrow graft donor. The analysis was used to quantify the total number of CD34+ cells in the product.

The method begins (Figure 1) with the identification and enumeration of the counting beads (A), which are conveniently visualized in a plot of fluorescence (StemCount) versus acquisition time. The time parameter allows perturbations in sample flow to be identified and eliminated by logical gating. The topmost example of Figure 1, there is a detectable interruption of event acquisition. 
These events acquired after the interruption proved to be no different than those acquire before and were therefore included. After logically gating the counting beads from the analysis, CD45 fluorescence is plotted versus side scatter and positive and dim events are identified and segregated from small debris and CD45 negative nucleated cells of the erythroid series. The count of events within this gate (B) is a preliminary step in the determination of CD45+ white blood cells (WBC). The last steps are determination of viability by 7AAD exclusion (E) and, a final cleanup on forward and side light scatter, with the events falling $(\mathrm{H})$ constituting the final CD45+ enumeration region. Note that there is little opportunity for error in the discrimination of cells and debris, since the great majority of debris was eliminated in the previous steps.

CD34 fluorescence intensity is plotted versus side scatter and CD34+ events are first identified among CD45+ events (C). CD34+ hematopoietic cells are known to have low side scatter. High side scatter events that are apparently $\mathrm{CD}_{34}+$ are present in the isoclonic or isotype control sample and are excluded from the analysis. The low side scatter CD34+ events are then checked for appropriate CD45 expression and side scatter properties (D). Another known artifact of bone marrow samples is a population of apparent $\mathrm{CD} 34+/ \mathrm{CD} 45$ bright cells of high light scatter. There are a small number of such events in the present example. Although not quantified in this analysis, two distinct CD34+ population can be discerned: one with dim CD45 expression and very low side scatter, and one with higher CD45 expression and relatively higher side scatter. As in $\mathrm{CD} 45^{+}$cells, the final cleanup for $\mathrm{CD} 45^{+/ C D} 34+$ events is $7 \mathrm{AAD}$ viability and appropriate light scatter. Early apoptotic cells that can still exclude 7AAD have low forward scatter and relatively high side scatter, and would not be included in $(\mathrm{H})$, the final CD34 enumeration region. The present sample has a few such cells.

It is worth mentioning that the College of American Pathologists requires that at least 100 cells be included in the final analytical region (12). This is because the precision of a rare event determination can be described by Poisson statistics, where the coefficient of variation (CV) of a count is directly proportional to the number of events counted. A count of 100 events yields a CV of 10\% (13), which CAP considers the lowest acceptable level of precision for a clinical assay. 
The second row of Figure 1 shows the same analysis performed on an isoclonic control. An isoclonic control is superior to the familiar isotype control and takes advantage of cold competition to distinguish between specific and nonspecific antibody binding. It includes PE-labeled antiCD34 (at the same concentration as the CD34 stained sample) in the presence of a large excess of unlabeled anti-CD34 antibody. Specific, but not nonspecific, binding of labeled antibody is competed by binding of the unlabeled antibody. In this example, FITC-labeled ant-CD45 and 7AAD have been included. This is important, because it permits us to use the identical gating strategy for experimental and control samples. Most of these events (either non-specific antibody binding or autofluorescence) are eliminated by the low scatter gate (C), all but 2 apparently CD34+ events are eliminated by the remaining cleanup gates.

The bottom 2 rows of Figure 1 show a hypothetical analysis using a simple, light scatter-first approach. In practice, we would not use this method in our clinical or research laboratory. The analysis begins by enumerating the beads (A) and limiting the analysis to viable cells (B). Applying the scatter-gate up front method is problematic: it is difficult to find a natural demarcation between cells and debris (C), possibly because of the presence of small CD45 negative nucleated erythroid cells of intermediate light scatter. Plotting CD34 versus CD45 (something we did not do in the ISHAGE analysis), allows us to easily distinguish CD45+ and dim events (D1 plus D2) from CD45 negative events (D3 plus D4), and allows us to identify CD45+/CD34+ cells either by the quadrant D2, or by the more stringent ovoid (E) region. The isoclonic control tells us that our strategy was not completely successful, revealing an obvious autofluorescent diagonal streak that passes through the D2 quadrant.

Examining the results tabulated in Figure 1 (and taking the ISHAGE protocol as the gold standard), we can see that the simple method underestimated the absolute CD45+ (WBC) count, and overestimated the CD34 count by $52 \%$ (quadrant method) or by $20 \%$ (tight ovoid region). We know that these results are suspect, since the isoclonic control (0.0\% and o events/ $\mu \mathrm{L}$ by the ISHAGE method) had absolute counts of 67 and 21 events/ $\mu \mathrm{L}$ for the quadrant and ovoid gates, respectively. 
4.1.4. Discussion/Conclusions. The sequential gating method first promoted by the ISHAGE strategy teaches several lessons that can be generalized to other applications: 1) A side scatter by fluorescence plot is often a far more effective way to begin an analysis than forward scatter by side scatter; 2) It is imperative to take advantage of known attributes of the cell population of interest, such as restricted expression of a marker (e.g. no authentic CD34+ hematopoietic progenitor cells are CD45 bright or have high side scatter), or absence of expression of a particular marker. In the present case, using CD19-PE-Texas Red would have allowed us to identify CD19+/CD34+ early B cells in bone marrow and remove them from the analysis. It also would have been helpful to remove autofluorescent events (i.e., cells fluorescing in the PE-Texas Red channel are either B cells or autofluorescent events); 3) The ISHAGE method also teaches us to do a final clean-up (viability and forward by side scatter gating) in the last steps of the analytical sequence, where it is easier to apply them with consistency. Although scatter gating need not always be performed last, it is much easier to apply after debris has been eliminated by some other method.

\subsection{Staining of Disaggregated Solid Tumors}

Solid tumors are complex and heterogeneous tissues often consisting of tumor cells spanning a wide variety of phenotypic variants, blood vessels, stroma and connective tissue, normal parenchymal cells and infiltrating immune cells. Often tumors have necrotic centers and are interspersed with hard scar tissue. Disaggregation into single cells for flow cytometry usually requires both mechanical and enzymatic dissociation that results in cell death and selection bias (discussed in detail in $(2,3)$ ). Dead cells, apoptotic cells and subcellular debris often comprise a significant proportion of events detected by flow cytometry. Although some can be eliminated by gating on forward by side scatter, many artifactual events remain, compromising analysis.

4.2.1. Data Analysis. In this example (Figure 2), we have used doublet discrimination, DNA content and an exclusion or dump gate to remove potential sources of artifact from a primary adenocarcinoma of the breast, in which we wished to measure coexpression of the mesenchymal/stem cell associated markers CD44 and CD90 on cytokeratin+ tumor cells. The top row of Figure 2 shows the analysis as we routinely perform it in our laboratory. We begin in histogram 1 with doublet discrimination which in this application uses a comparison of forward 
the default forward scatter pulse signal (height or area depending on instrumentation) and forward scatter pulse width (time of flight) to identify and remove events with pulse-width too great for the pulse height or area. These are cell doublets, undigested cell clusters, or single events that were too closely spaced in the sample stream to be discriminated by pulse threshold. In the present sample such clusters constituted $23 \%$ of measured events. In histogram 2, we examine DNA content as measured by DAPI binding. In the present analysis we are also looking at intracellular cytokeratin so the cells were permeabilized after surface staining and fixation allowing anti-cytokeratin (and DAPI) to enter the cells. In the absence of permeabilization, DAPI can be used as a viability exclusion dye like 7AAD (Figure 1). Plotting DAPI fluorescence by forward scatter, we are able to gate on events having at least a full complement of DNA compared to tumor infiltrating lymphocytes (which are identified in histogram 4 as CD45 bright). In the present example $65 \%$ of measured events had less than a full complement of DNA. In histogram 3 we either leave the scatter gate $\mathrm{C}$ wide open (top panel) or use it to remove low light scatter events that appear when DNA gating is not used (middle and bottom panel). Histogram 4 gates singlet events with DNA content $\geq 2 \mathrm{~N}$ for absence of expression of CD45 and a cocktail of hematopoietic lineage markers. This removes tumor infiltrating immune cells from the analysis, including myeloid cells that are cytokeratin + because they have ingested tumor cells or tumor cell fragments. In histogram 5 we gate on cytokeratin+ high side scatter cells, on which we measure coexpression of CD44 and CD9o (histogram 6). The middle panel shows what happens when doublet discrimination and DNA gating is eliminated, and sequential gating begins with forward and side light scatter. Autofluorescent events appear as a diagonal streak in histogram 4, the resolution of cytokeratin events is diminished (histogram 5), and CD44+ cells are underrepresented (histogram 6). The bottom panel shows what happens when no strategies to remove artifact, including the use of a CD45/heme lineage dump gate, are employed. The resolution of cytokeratin events is further diminished (histogram 5), and the expression of CD44 and CD90 are obscured by autofluorescent events (histogram 6).

4.2.2. Flow minus outcomes (FMOplus). Exactly what constitutes the proper staining controls for multicolor assays is still controversial. In example 4.1, an FDA approved in vitro diagnostic assay, there were no control tubes for $\mathrm{CD} 45$ or $7 \mathrm{AAD}$ staining, but there was an isoclonic control for 
CD34 staining, the critical outcome measurement. The purpose of this control is two-fold: 1) to guide $\mathrm{CD} 34+$ region placement; 2) to ensure that proper spectral compensation was performed (i.e. if CD45-FITC staining were spilling over into the PE detection channel, the isoclonic control would have read as positive). In a 3-color example such as 4.1, mistakes in spectral compensation are unlikely, but in multi-color assays, it is necessary to rigorously control for the effects of spillover, especially where tandem dyes are concerned. It is for this reason that a series of Fluorescence Minus One (FMO) controls have been advocated (14). According to this approach, a 5-color assay should have one experimental tube in which all fluorochromes are included, and a series of 5 FMO control tubes in which fluorochromes are systematically eliminated, one at a time. This is an ideal situation, but it is costly in terms of sample, reagents and instrument time. A middle ground can be reached by running all FMO controls during assay development, but considering the way in which the data will be analyzed to select essential controls once the assay has been finalized and is in routine use. Toward this end, it is helpful to distinguish between variables used for artifact removal, classifying variables and outcome variables $(2,3)$. In 4.2.1 the principal objective was to measure CD44 and CD9o expression on non-hematopoietic, cytokeratin + tumor cells. Therefore, doublet discrimination DAPI, CD45, and the lineage cocktail are artifact removal variables, cytokeratin and side scatter are the classifiers used to define the population of interest (epithelial tumor cells), whereas CD44 and CD9o could be considered as outcomes measured on cytokeratin+ tumor cells. During assay development, a complete series of FMO controls would guide placement of artifact removal and classifier regions, as well as outcomes, permitting validation of multicolor spectral compensation as well. Controls for artifact removal and classifier variables that prove necessary to make objective gating decisions could be retained. For outcome variables a single control tube, eliminating antibodies for all outcome variables, but retaining those required as classifiers, is sufficient. We have termed this a Fluorescence Minus Outcomes (FMOplus) control. Figure 3 illustrates the use of an FMOplus control for the staining tube shown in 4.2.1. All of the reagents required to define the classifier are present, but anti-CD44 and anti-CD9o have been omitted, permitting precise placement of the outcome regions. It is worth noting that experience during assay development with FMO controls 
and samples without cytokeratin+ cells, allows us to be confident about the placement of lineagenegative and cytokeratin classifier gates.

4.2.3. Sequential gating versus light scatter gating. What happens when gate on light scatter instead of following the procedures outlined for artifact removal, is shown in the second row of Figure 2. Removal of heme-lineage positive events does much to clean up the sample, but leaves us underestimating the proportion of cytokeratin+ tumor cells and their CD44+/CD90- subset. The third row of figure 2 stays with conventional side scatter gating but dispenses with the hemelineage dump gate. This is a total disaster, as the 6-fold overestimation of the proportion of CD44+/CD90+ cells among cytokeratin+ tumor cells attests. In the rightmost histogram, an autofluorescent diagonal streak can be seen in quadrant F2 and an entirely spurious population of CD44 bright CD90 \pm events can be seen spanning F2 and F4.

4.2.4. Discussion/Conclusions. In Figure 2 we used forward scatter pulse analysis to eliminate cell clusters (a frequent issue in digested solid tissues), and removed events with hypodiploid DNA and hematopoietic cells from our analysis prior to measuring CD44 and CD9o expression on cytokeratin+ tumor cells. We compared this strategy to conventional light scatter-based gating, with and without the heme-lineage dump gate. We found that the use of doublet discrimination and DNA gating significantly increased the signal to noise in the identification of cytokeratin+ and CD44+/CD90+ events, as evidenced by tighter positive populations with more separation from negative events. It also decreased the proportion of cytokeratin+ events among heme-lineage negative events, and increased the proportion of CD44+/CD90- events among cytokeratin+ events. Reliance on a light scatter gate alone as a denominator upon which to measure cytokeratin + tumor cells was entirely unsatisfactory, and resulted in the inclusion of demonstrably autofluorescent events (recognized by linear correlation of fluorescence in the PE and PE-Texas Red channels), and obfuscation of cell populations that are clearly demarcated in multidimensional space, when proper artifact removal is performed.

The notion that measured variables can be divided into those used to remove artifact, those used to classify population(s) of interest and those that are outcomes measured on populations of interest is somewhat fluid: you can measure interferon production (outcome) on CD8 T cells (classifier), or you can gate on cells producing interferon (classifier) and determine their 
phenotype(s) (outcomes). Nevertheless, this approach creates a hierarchy that simplifies data display and specifies that the essential FMOplus control be prepared for the outcome variable(s).

\subsection{Expression of mesenchymal markers on adipose stromal vascular cells}

4.3.1. Panel design. Adipose is a dynamic tissue that is richly vascularized. Associated with the small vessels are several distinct populations of adult mesenchymal and endothelial stem cells (15). The purpose of this panel was to identify the known progenitor populations in the stromal vascular fraction of human adipose tissue and characterize their expression of the mesenchymal markers CD73 (Ecto-5'-nucleotidase), CD90 (Thy-1) and CD105 (Endoglin). The major challenge in panel design is the violent blue-excited fluorescence in the channels used for FITC, PE and PE-Texas Red (ECD) detection (16). One possible solution (unavailable to us at the time of panel design) is to eliminate FITC, which can only be excited by the $488 \mathrm{~nm}$ laser, and use a yellow-green $(561 \mathrm{~nm})$ or green (532 $\mathrm{nm}$ ) laser to excite PE and the PE-tandem dyes.

4.3.2. Artifact removal. In Figure 4 we combined the artifact removal strategies used in Figures 1 - 3 . A plot of time $v s$ log side scatter was used to detect fluidic disturbances (none were present here); doublet discrimination (A) and DNA content (B) were used to identify single cells with a full complement of DNA; CD45 bright lymphocytes (K) served as an internal standard for diploid DNA content and a landmark for light scatter (D).

4.3.2.1. Removal of autofluorescent events. Cellular autofluorescence is due to expression of highly fluorescent biomolecules such as NADH, FAD, riboflavin, flavin coenzymes and lipofuscins (8). Cellular autofluorescence has been attributed to metabolically active mitochondria which provide the energy required to execute the final steps of apoptosis (17). Broadly autofluorescent events can be recognized as diagonal streaks across bivariate histograms of the channels in which they emit. We exploited this property to remove them from the analysis. First we plotted FITC vs PE, FITC vs PE-Texas Red and PE vs PE-Texas Red, drawing a region on the diagonal streak in each histogram (Figure 4). Events falling within a compound Boolean gate including only events present in all three regions, were designated autofluorescent and eliminated from subsequent analyses. In the present example, autofluorescent events comprised $3.1 \%$ of total events (I), and had high side scatter compared to the majority of cells and low forward scatter compared to lymphocytes, a profile 
consistent with early apoptotic cells. The FITC, PE, ECD and scatter profiles of the cells that remain after autofluorescent events have been removed are shown in Figure 5. Because autofluorescence is defined in three dimensions, the rectilinear edges of regions $\mathrm{F}, \mathrm{G}$ and $\mathrm{H}$ do not impinge on the populations defined as Not AutoFl in this analysis. In fact, a major population of $\mathrm{CD}_{105}+/ \mathrm{CD} 146+$ cells lies directly in the path of region $\mathrm{G}$, but was not eliminated because the events fall outside of the $\mathrm{F}$ and/or $\mathrm{H}$ regions.

The present approach to autofluorescence is a risky exercise if most of the cells of interest are also highly autofluorescent. It can also underestimate triple positive events, which may spill into the autofluorescent gate. In the present case removal of interfering autofluorescent cells cleaned up the analysis without eliminating cells of interest.

4.3.2.2. Dump gate. In Figure 2 we saw that the dump gate is an essential part of the cleanup strategy. Here we used CD45 and a lineage cocktail consisting of CD14/CD33 and glycophorin-A/CD235a to remove hematopoietic cells and other bad actors (J). Note that we used PECy5 for our lineage cocktail. Ordinarily, using PECy5 and APC in the same panel is a bad idea, unless marker expression is mutually exclusive. The reason is that PECy5 is excited by the blue laser, and the Cy5 portion is excited again by the red laser, emitting with a pattern indistinguishable from red-excited APC. In the present case we are safe, because PECy5+ events are eliminated from the analysis.

4.3.3. Classifiers and outcomes. CD31, CD34 and CD146 comprise our classifier variables, defining pericytes (CD146+/CD31-), supra-adventitial adipose stromal cells (CD34+/CD31-/CD146-) and

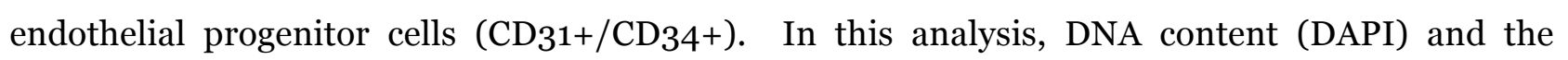
mesenchymal markers CD73, CD90 and CD105 served as outcome variables. Some of the classifier markers (CD34, CD146) also doubled as outcomes, further characterizing the classifier population (e.g. CD146 expression on endothelial progenitor cells) or defining subpopulations within a classifier population (a minor $\mathrm{CD}_{34}+$ subpopulation within pericytes). In this sample all three stem cell populations expressed CD90; CD73 expression was limited to supra-adventitial adipose stromal cells and to a lesser extent to endothelial progenitor cells. CD105 was expressed exclusively on endothelial progenitor cells, which also had the highest fraction of proliferating cells (DNA content $>2 \mathrm{~N})$. 
4.3.4. Discussion/Conclusions. Enzymatic digestion and mechanical disaggregation are necessary for preparing adipose tissue for analytic flow cytometry or cell sorting. Unfortunately, they result in a variety of artifacts, some of which can be addressed and corrected during the analysis. Overdigestion can kill cells, damage antigenic epitopes, and release DNA that traps viable cells. Underdigestion results in clusters of adherent cells. Nonadherent or loosely adherent cells (i.e. immune cells) are preferentially recovered, whereas fragile or tightly adherent cells are underrepresented. Further, subcellular debris can bind antibody and be misclassified as cells; apoptotic and necrotic cells have aberrant and unpredictable antigen expression. Some of these problems can be mitigated by careful tissue preparation. The inclusion of DNase in the digestion step can reduce cell clumping and increase cell yield and debris. Dead cells and debris can be reduced with the use of a Ficoll/Hypaque density gradient as a final cleanup step. Even under the best of circumstances, a significant proportion of acquired events are not single cells in good health at the time of staining and fixation and must be identified as such during data analysis.

Acknowledgements. The authors would like to E. Michael Meyer and Melanie Pfeifer for their excellent technical assistance, and Linda Moore, MT (ASCP), Supervisor of the University of Pittsburgh Medical Center Adult Hematopoietic Stem Cell Laboratory, Adam M. Brufsky MD, PhD, Co-Director UPMC Comprehensive Breast Cancer Center, James D. Luketich, MD, Chair Department of Cardiothoracic Surgery, and J. Peter Rubin MD, Chair of Plastic Surgery for providing the human tissue specimens used in this report. The University of Pittsburgh Cytometry Laboratory is supported by Cancer Center Support Grant P30CA047904, Breast cancer studies were supported by grants from the Department of Defense (BCo32981, BCo44784, BC132245 and BC113183) and the Glimmer of Hope Foundation. Adipose studies were supported by the Department of Defense (W81 XWH-o8-2-0032) and the National Institutes of Health (RO1-CA114246-08, R21 CA191647). The authors declare there are no conflicts of interest in this article.

\section{Figure Legends}

Figure 1. ISHAGE versus simple method for absolute CD34 count. The top 2 panels illustrate the ISHAGE method of CD34 determination as modified by the University of Pittsburgh Hematopoietic Stem Cell Laboratory. All gating is sequential with Boolean logic as indicated in the histogram titles. ISHAGE method, from left to right: Beads (A) are identified versus acquisition time (note that acquisition was briefly interrupted. without anv apparent disturbance): CD45+ events (B) are isolated from CD34 negative cells 
(nucleated erythroid) and debris; Low light scatter CD34+ events are identified (D), Note: 2 distinct scatter populations characteristic of BM CD34+ cells); Viable (7AAD excluding) CD45+ cells (E) are identified; The final CD45 enumeration gate (F) excludes apoptotic cells with low forward scatter and high side scatter; Viable CD34+ cells (G) are identified; The final CD34 enumeration gate (H). Simple method, from left to right: Beads (A) are identified versus acquisition time; Viable cells (B) are identified among total events; Viable CD34+ events are identified in a quadrant (D2) and by a tight gate (E).

Figure 2. Detection of CD44+/CD90+ mesenchymal breast cancer cells in primary adenocarcinoma of the breast, with and without artifact removal. All gating is sequential with Boolean logic as indicated in the histogram titles. The top row shows our standard protocol for artifact removal prior to detection of CD44 and CD9o subpopulations of epithelial tumor cells in breast cancer. The panels (left to right) show the steps in artifact removal: 1) Eliminate doublets and cell clusters by forward scatter pulse analysis; 2) Eliminate events with hypodiploid DNA or no detectable DNA; 3) Check scatter properties; 4) Use a dump gate to eliminate cells that stain for markers not present on the population of interest (CD45, CD14, CD33 and GlycophorinA/CD235a); 5) Identify epithelial tumor cells by high side scatter and bright cytokeratin staining; 6) Determine the degree of CD44 and CD90 expression on cytokeratin+ tumor cells. Only a minority of cytokeratin+ tumor cells co-express CD44 and CD90, antigens often present on mesenchymal tumorigenic cells (1.2\% of cytokeratin+ events). The second row displays what happens when cell clusters and hypodiploid events (gate A and B) are not eliminated. The bottom row shows the staining of all scatter-gated events without any artifact removal and is closest to conventional analysis as practiced on peripheral blood mononuclear cells.

Figure 3. The use of a Fluorescence Minus Outcomes (FMOplus) control to guide the placement of regions defining CD44+ and CD90+ events. Histograms in the leftmost box permit artifact removal as in Figure 2. The population of interest (epithelial tumor cells) is defined by the classifier cytokeratin and side scatter (center box). The rightmost box shows expression of the outcome markers (CD44 and CD90) on cytokeratin+ cells (top) and the FMOplus control used to set the quadrants. Note that these lung cancer cells are CD9o negative, and that CD44 expression forms a continuum with no clear demarcation between positive and negative events. Objective determination of this region would have been impossible without an appropriate control.

Figure 4. Detection of mesenchymal markers on progenitor populations within the adipose stromal vascular fraction. The top two rows illustrate the process of artifact removal, similar to figures 2 and 3, but with addition of the time parameter to detect fluidic problems and compound Boolean gating to eliminate autofluorescent events. The two center histograms are intermediate to the definition of the classifier populations (pericytes, supra-adventitial adipose stromal cells, and endothelial progenitor cells). The bottom nine histograms show the expression of mesenchymal markers CD73, CD9o and CD105 and DNA ploidy on the classifier populations. CD34 and CD146 expression is also shown for classifier populations that do not include these markers in their definition.

Figure 5. Characterization of the Not AutoFl population. These histograms illustrate what remains when the autofluorescent cells were removed from the data shown in Figure 4. Note that CD105+/CD146+ cells were not eliminated because they fall outside of the $\mathrm{F}$ and/or $\mathrm{H}$ regions.

\section{References}

1. Donnenberg, V. S., Meyer, E. M., and Donnenberg, A. D. (2009) Measurement of Multiple Drug Resistance Transporter Activity in Putative Cancer Stem/Progenitor Cells. In Methods in Molecular Biology (Yu, J., ed) Vol. 568 Cancer Stem Cells pp. 261-279, Humana Press, Springer, New York

2. Donnenberg, V. S., Landreneau, R. J., Pfeifer, M. E., and Donnenberg, A. D. (2013) Flow cytometric determination of stem/progenitor content in epithelial tissues: an example from nonsmall lung cancer and normal luna Cvtometrv A 83 141-149 
3. Zimmerlin, L., Donnenberg, V. S., and Donnenberg, A. D. (2011) Rare Event Detection and Analysis in Flow Cytometry: Bone marrow mesenchymal stem cells, breast cancer stem/progenitor cells in malignant effusions, and pericytes in disaggregated adipose tissue. In Flow Cytometry Protocols (Hawley, T. S., and Hawley, R. G., eds) Vol. 699 pp. 251-273, Humana Press, New York, N.Y.

4. Zimmerlin, L., Donnenberg, V. S., Pfeifer, M. E., Meyer, E. M., Peault, B., Rubin, J. P., and Donnenberg, A. D. (2010) Stromal vascular progenitors in adult human adipose tissue. Cytometry $A$ 77A, 22-30

5. Donnenberg, V. S., Landreneau, R. J., and Donnenberg, A. D. (2007) Tumorigenic stem and progenitor cells: Implications for the therapeutic index of anti-cancer agents. $J$ Control Release 122, 385-391

6. Mandy, F. F., Nicholson, J. K. A., and McDougal, J. S. (2003) CDC Guidelines for Performing SinglePlatform Absolute CD4+ T-Cell Determinations with CD45 Gating for Persons Infected with Human Immunodeficiency Virus. Morbidity and Mortality Weekly Report 52, 1-13

7. Siena, S., Schiavo, R., Pedrazzoli, P., and Carlo-Stella, C. (2000) Therapeutic Relevance of CD34 Cell Dose in Blood Cell Transplantation for Cancer Therapy. Journal of Clinical Oncology 18, 1360-1377

8. Aubin, J. E. (1979) Autofluorescence of viable cultured mammalian cells. J Histochem Cytochem 27 , $36-43$

9. Gratama, J. W., Kraan, J., Keeney, M., Sutherland, D. R., Granger, V., and Barnett, D. (2003) Validation of the single-platform ISHAGE method for CD34+ hematopoietic stem and progenitor cell enumeration in an international multicenter study. Cytotherapy 5, 55-65

10. Sutherland, D. R., Nayyar, R., Acton, E., Giftakis, A., Dean, S., and Mosiman, V. L. (2009) Comparison of two single-platform ISHAGE-based CD34 enumeration protocols on BD FACSCalibur and FACSCanto flow cytometers. Cytotherapy 11, 595-605

11. Sutherland, D. R., Anderson, L., Keeney, M., Nayar, R., and Chin-Yee, I. (1996) The ISHAGE guidelines for CD34+ cell determination by flow cytometry. International Society of Hematotherapy and Graft Engineering. J Hematother 5, 213-226

12. College_of_American_Pathologists_Commission_on_Laboratory_Accreditation. (2007) Flow Cytometry Checklist. Vol. FLO.30585 pp. 1-57, College of American Pathologists

13. Shapiro, H. M. (2003) Practical flow cytometry, Wiley-Liss, Hoboken, N.J.

14. Roederer, M. (2001) Spectral compensation for flow cytometry: visualization artifacts, limitations, and caveats. Cytometry 45, 194-205

15. Zimmerlin, L., Donnenberg, V. S., Rubin, J. P., and Donnenberg, A. D. (2013) Mesenchymal markers on human adipose stem/progenitor cells. Cytometry Part A 83A, 134-140

16. Katz, A. J., Tholpady, A., Tholpady, S. S., Shang, H., and Ogle, R. C. (2005) Cell Surface and Transcriptional Characterization of Human Adipose-Derived Adherent Stromal (hADAS) Cells. STEM CELLS 23, 412-423

17. Levitt, J. M., Baldwin, A., Papadakis, A., Puri, S., Xylas, J., Münger, K., and Georgakoudi, I. (2006) Intrinsic fluorescence and redox changes associated with apoptosis of primary human epithelial cells. Journal of biomedical optics 11, 064012-064012-064010 


\section{Artifact Removal:}

Fluidic disturbance

Cell clusters

Debris and hypodiploid cells

Autofluorescent events

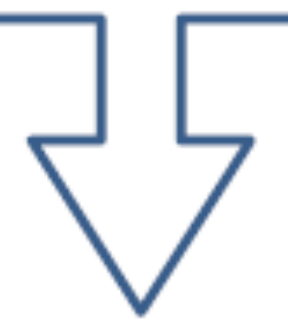

Hierarchical Definition of Classifier populations

Measurement of Outcomes on Classifier populations 
CD45-FITC, CD34-PE, 7AAD, StemCount Beads (ISHAGE)
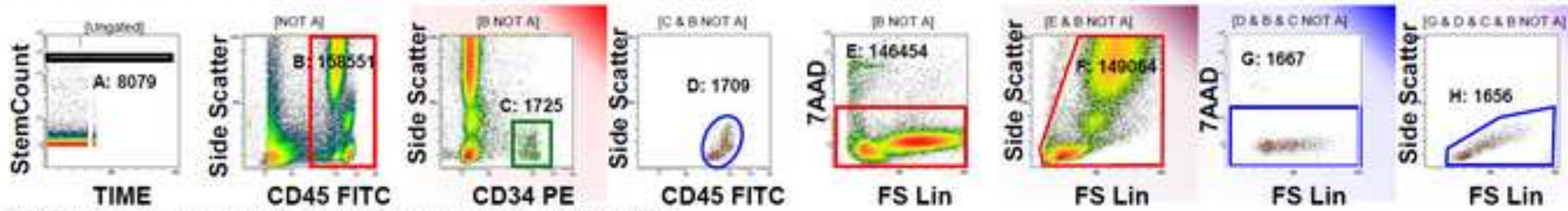

CD45-FITC, Isoclonic control-PE, 7AAD, StemCount Beads (ISHAGE)
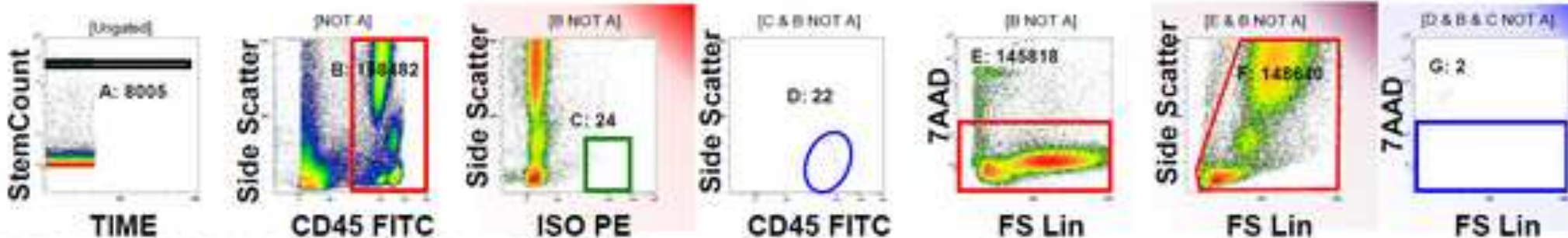

FS Lin

FS Lin

CD4S-FITC, CD34-PE, 7AAD, StemCount Beads (SIMPLE)
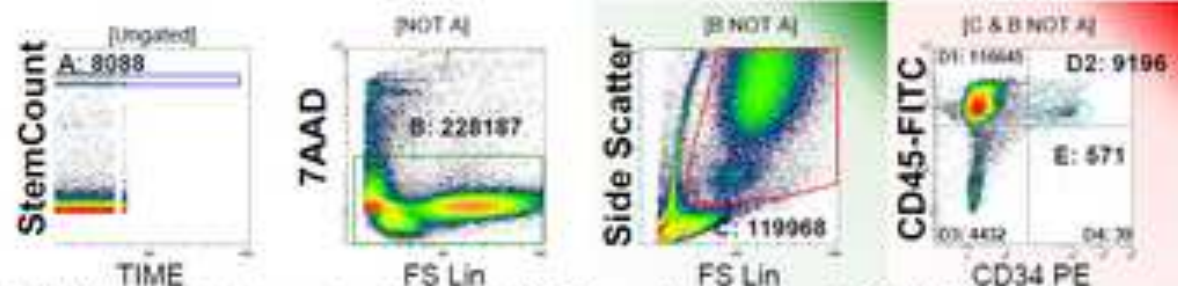

CD45.FiTC, Isoclonic control.PE, 7AAD, Stem Count Beads (SIMPLE)
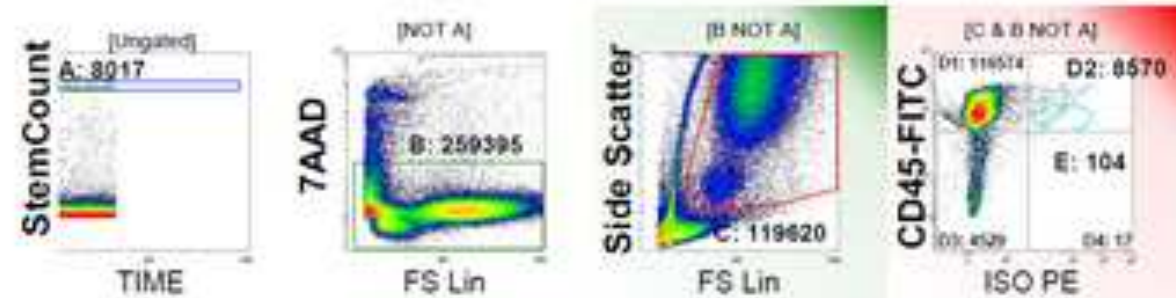

\begin{tabular}{|c|c|c|c|c|c|c|c|}
\hline & & \multicolumn{2}{|c|}{ ISHAGE } & \multicolumn{2}{|c|}{ Simple (Quad) } & \multicolumn{2}{|c|}{ Simple (Tight) } \\
\hline & & Percent & Cells/ui. & Percent & Cells/me & Percent & Cells/ul \\
\hline \multirow{2}{*}{ Full Stain } & $\mathrm{CD} 45$ & & 18,451 & & 15,559 & & \\
\hline & $\operatorname{CO} 34$ & $1.1 \%$ & 205 & $7.3 \%$ & 1,137 & $0.5 \%$ & 71 \\
\hline \multirow{2}{*}{ Isoclonic } & $\mathrm{CO} 45$ & & 18,568 & & 15,610 & & \\
\hline & $\operatorname{CD} 34$ & $0.0 \%$ & 0 & $6.8 \%$ & 1,069 & $0.1 \%$ & 13 \\
\hline
\end{tabular}

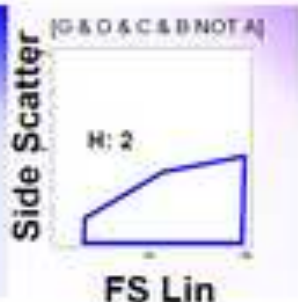

FS Lin 
Artifact Removal: Analysis limited to singlets, diploid and heme-lineage negative events

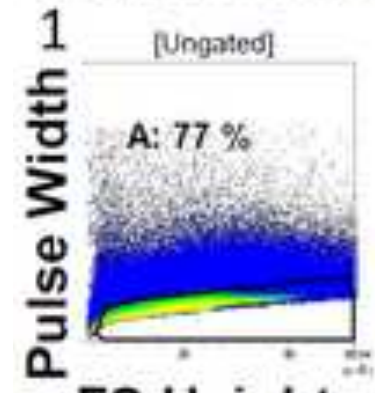

FS Height

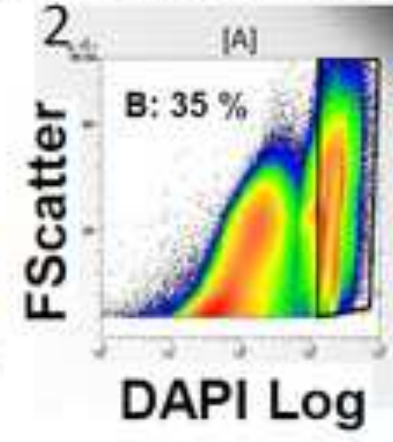

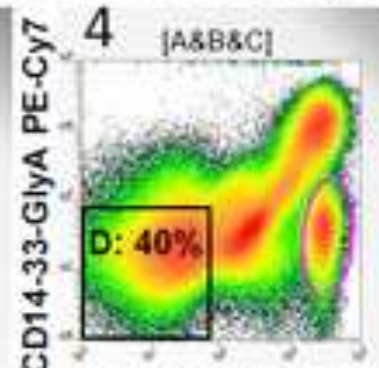

CD45 APC-Cy7 iCTK FITC

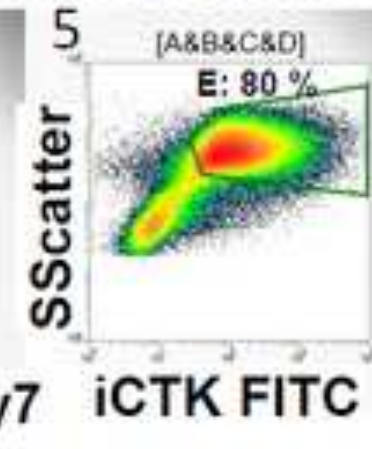

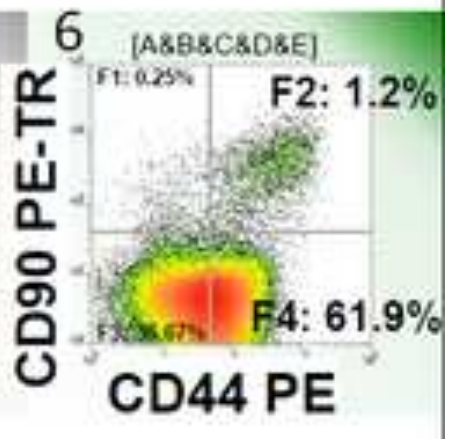

Artifact Removal: Analysis limited to heme-lineage negative events

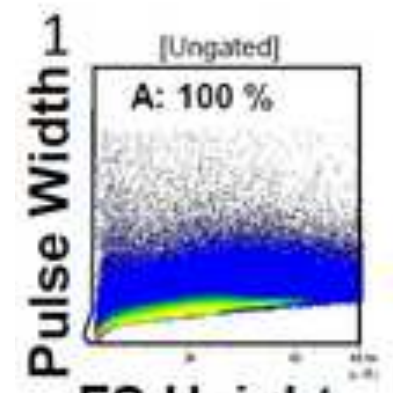

FS Height

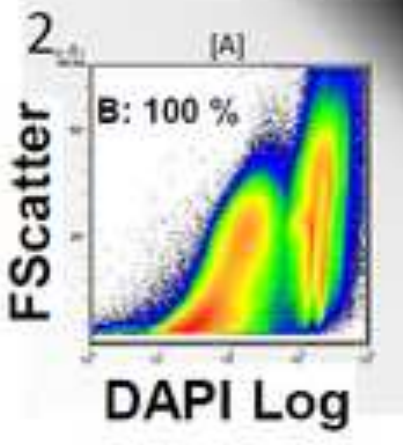

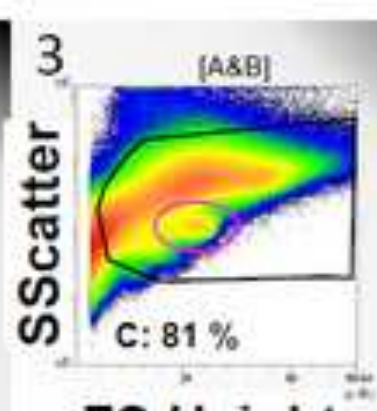

FS Height

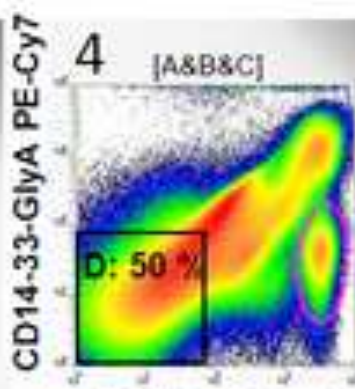

CD45 APC-Cy7 iCTK FITC
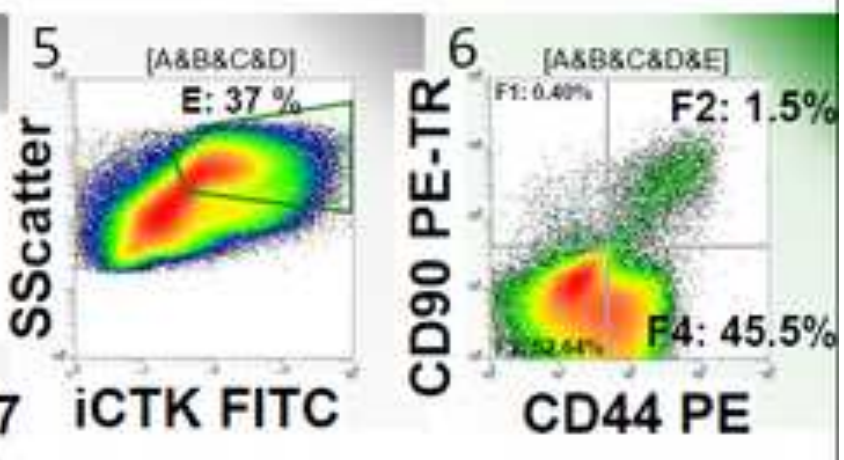

BrCATSC022. Tumor

No Artifact Removal: Analysis includes all scatter-gated events

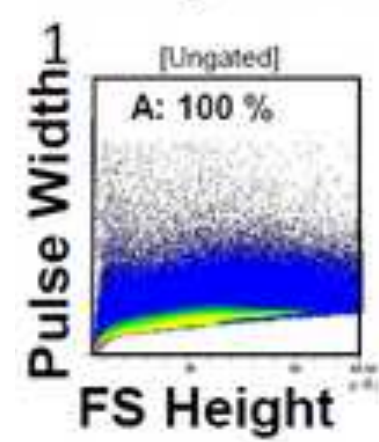

FS Height

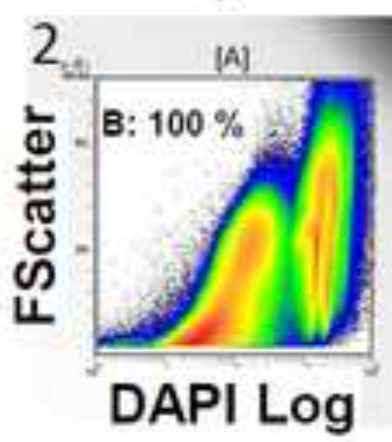

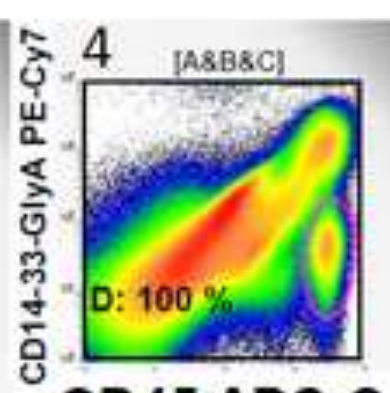

CD45 APC-Cy
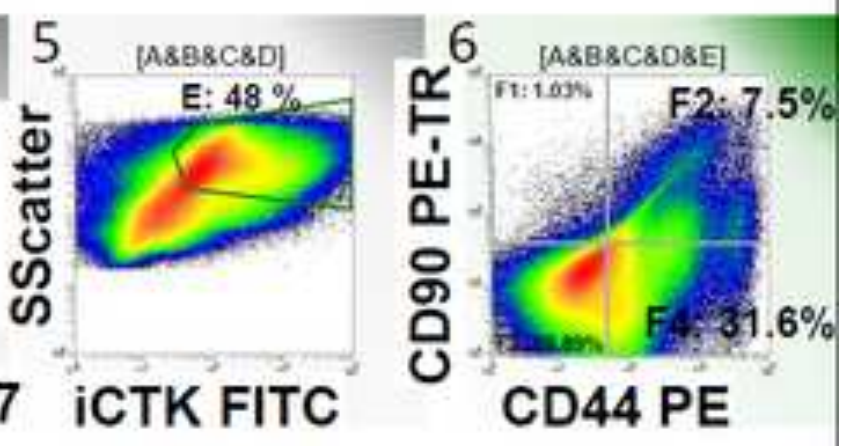
Artifact Removal: Analysis limited to singlets, $D N A \geq 2 N$ and heme-lineage negative events

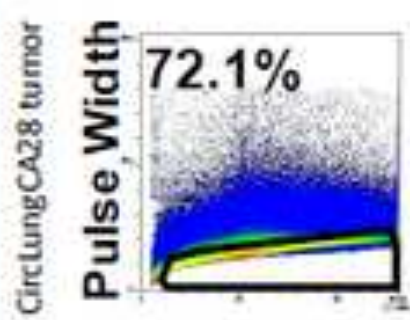

FS

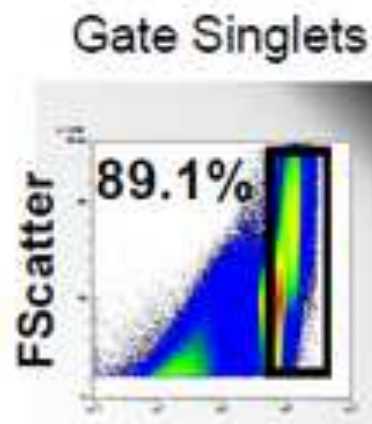

DAPI Log

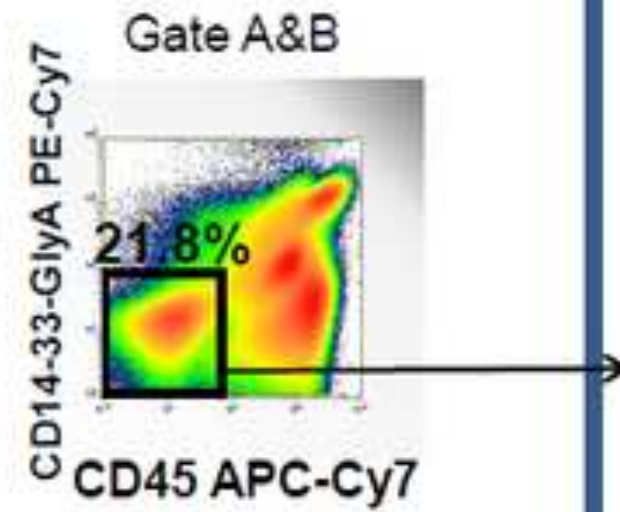

O CD45 APC-Cy7
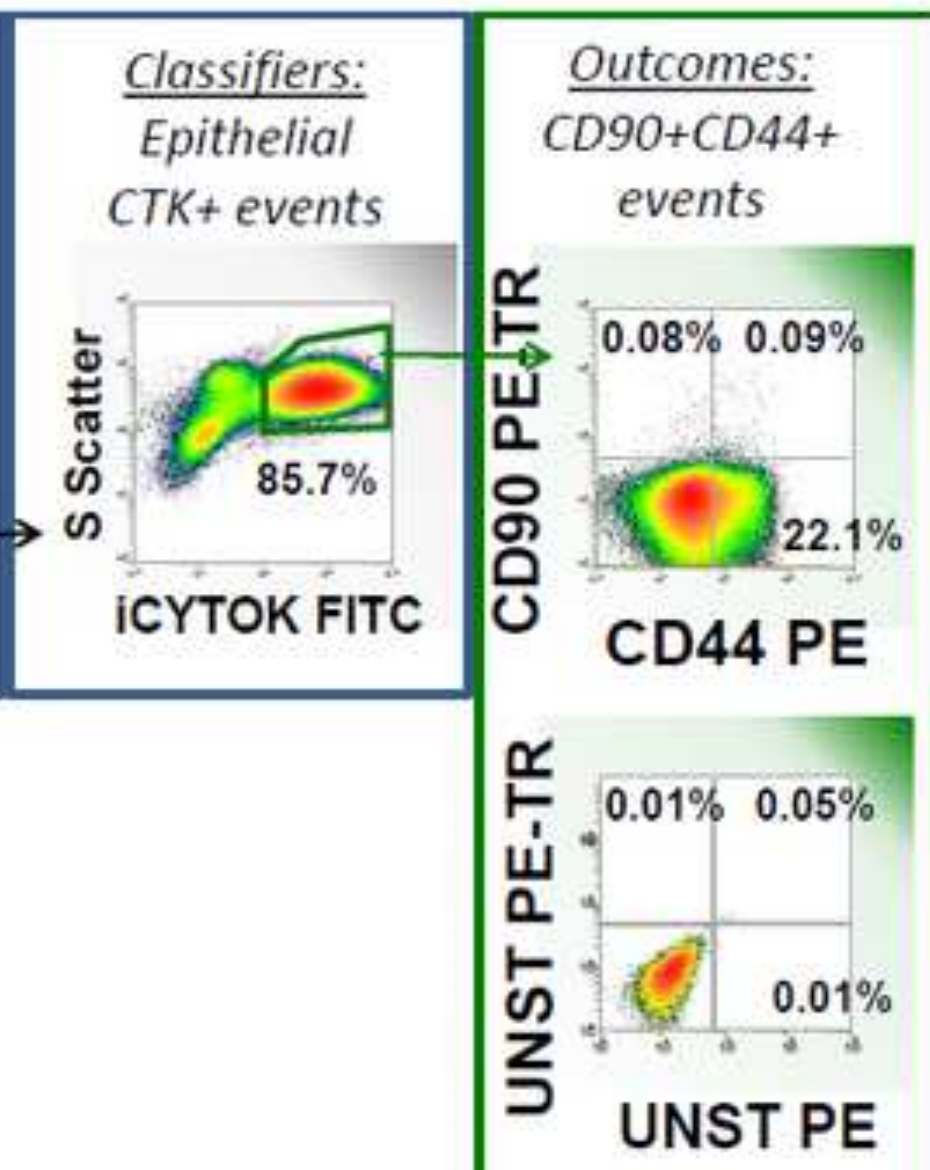

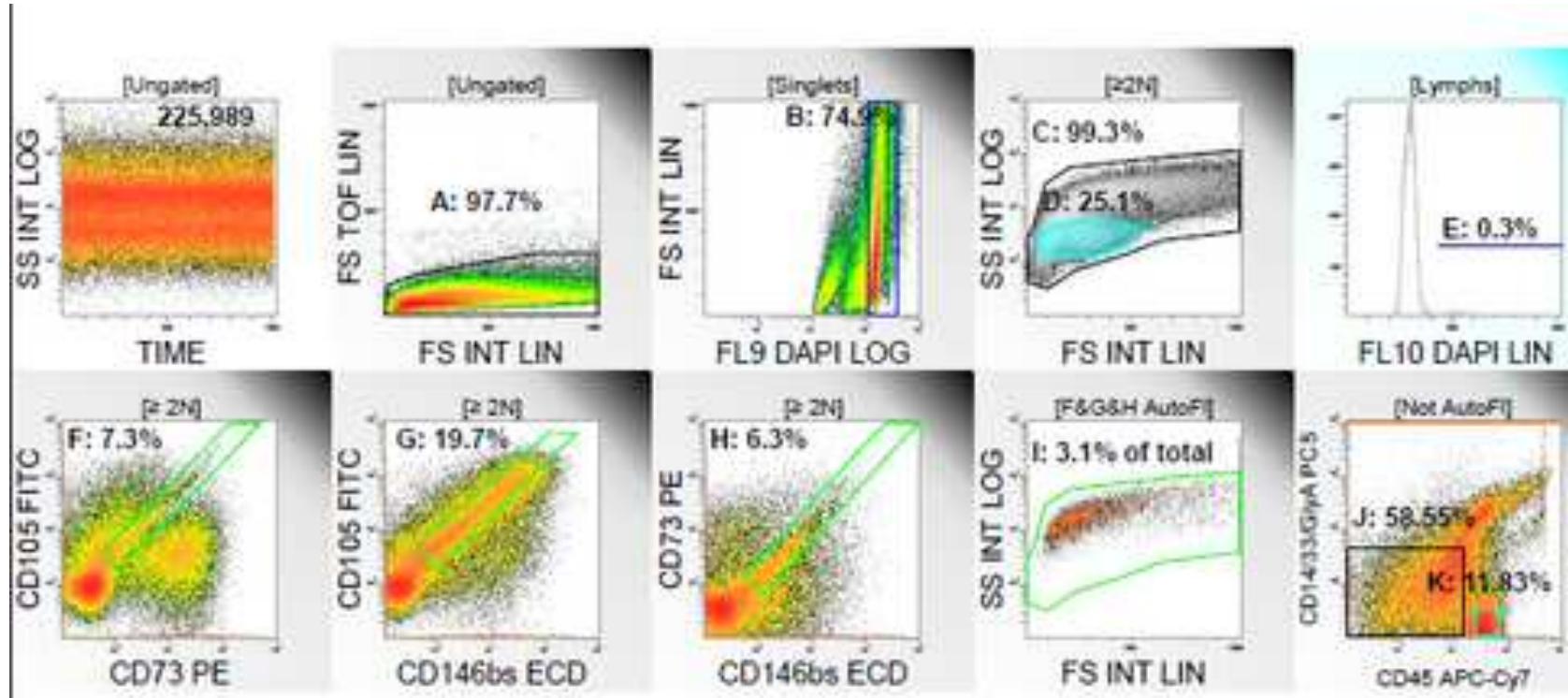

FATsC 08_28_14
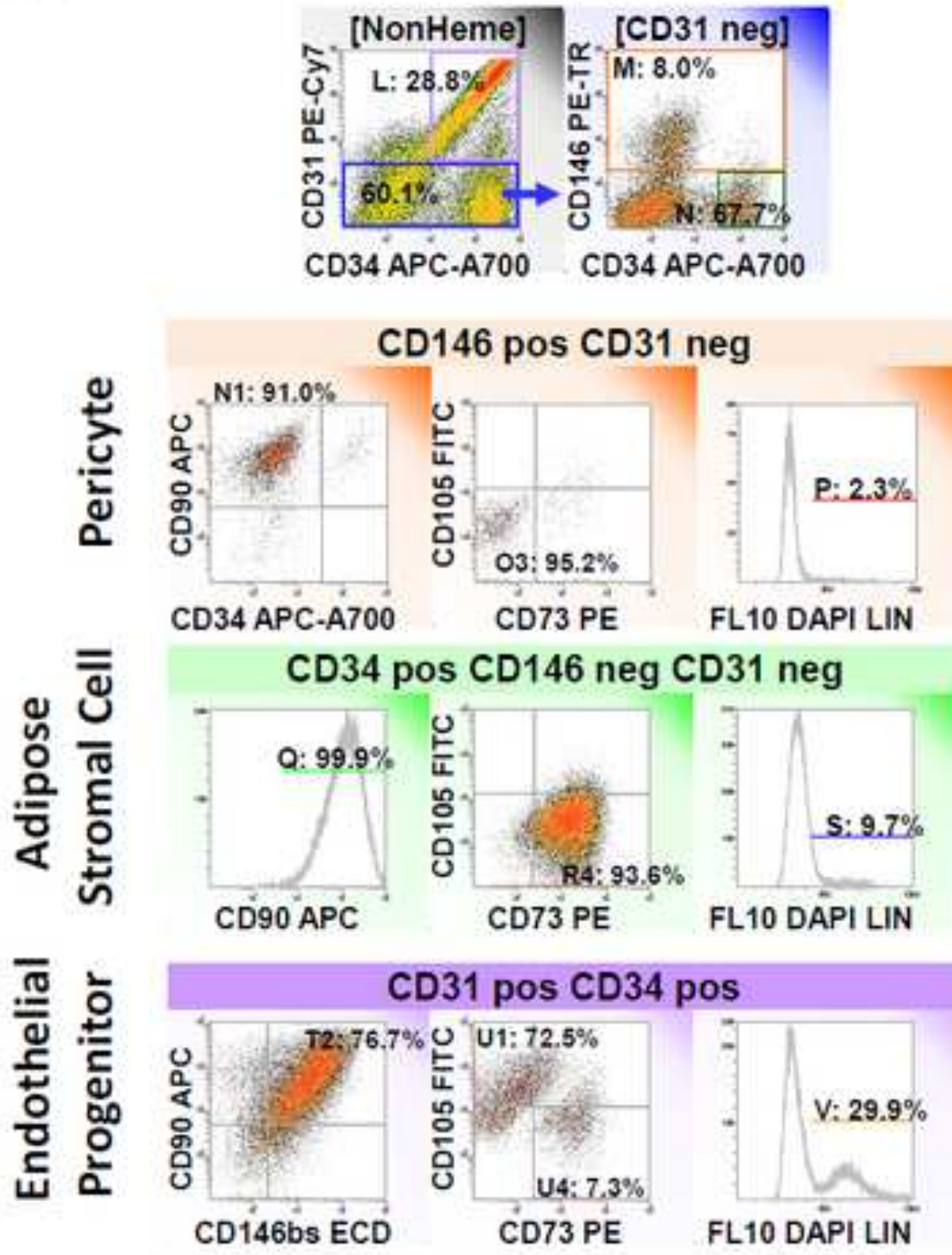
Before removal of autofluorescent events
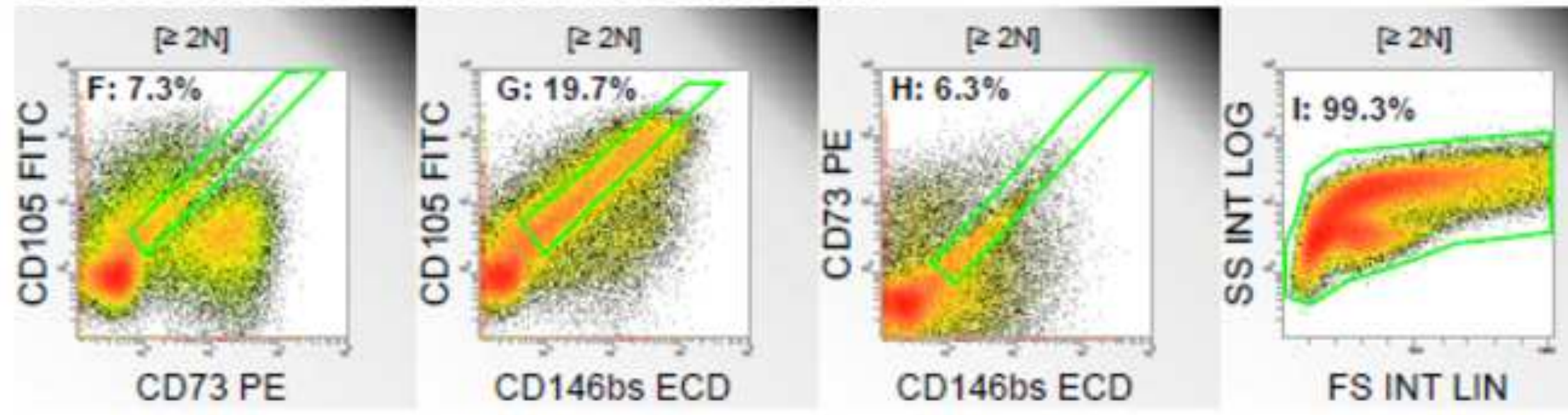

\section{After removal of autofluorescent events}
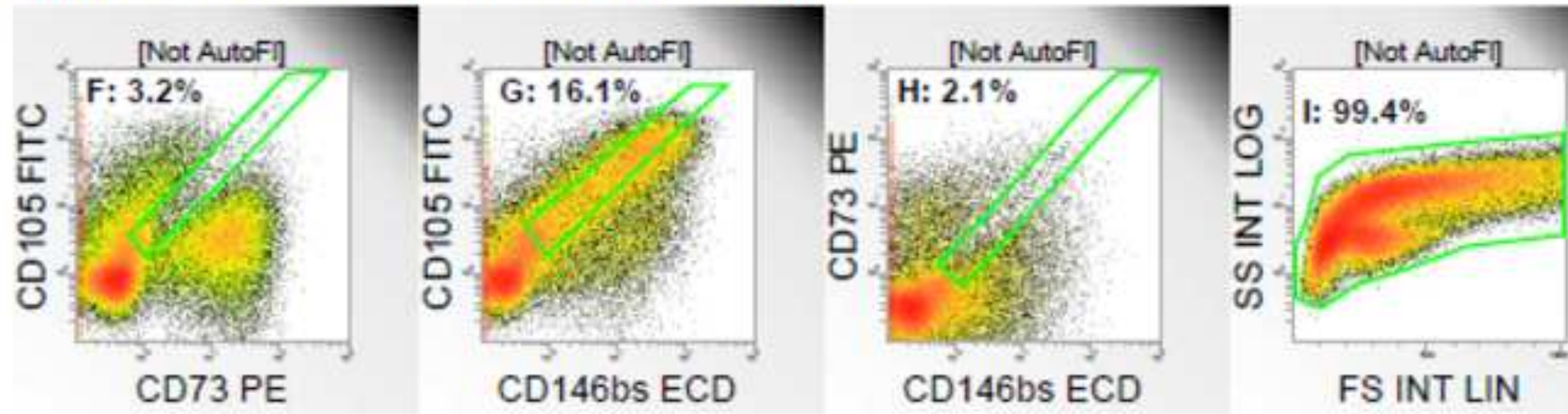

FATSC 08_28_14 